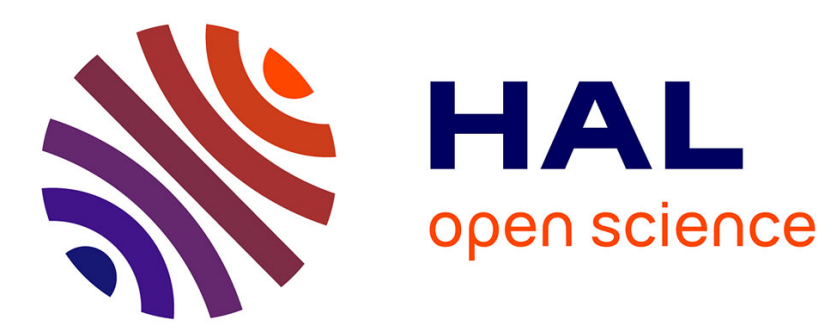

\title{
M\&S Support for Crisis and Disaster Management Processes and Climate Change Implications
}

\author{
Orlin Nikolov, Nikolay Tomov, Irena Nikolova
}

\section{To cite this version:}

Orlin Nikolov, Nikolay Tomov, Irena Nikolova. M\&S Support for Crisis and Disaster Management Processes and Climate Change Implications. 1st International Conference on Information Technology in Disaster Risk Reduction (ITDRR), Nov 2016, Sofia, Bulgaria. pp.240-253, 10.1007/978-3-31968486-4_19. hal-03213131

\section{HAL Id: hal-03213131 \\ https://hal.inria.fr/hal-03213131}

Submitted on 30 Apr 2021

HAL is a multi-disciplinary open access archive for the deposit and dissemination of scientific research documents, whether they are published or not. The documents may come from teaching and research institutions in France or abroad, or from public or private research centers.
L'archive ouverte pluridisciplinaire HAL, est destinée au dépôt et à la diffusion de documents scientifiques de niveau recherche, publiés ou non, émanant des établissements d'enseignement et de recherche français ou étrangers, des laboratoires publics ou privés. 


\title{
M\&S Support for Crisis and Disaster Management Processes and Climate Change Implications
}

\author{
Orlin Nikolov ${ }^{1}$, Nikolay Tomov ${ }^{2}$ and Irena Nikolova ${ }^{3}$ \\ ${ }^{1}$ Crisis Management and Disaster Response Centre of Excellence, Sofia, Bulgaria \\ orlin.nikolovecmdrcoe.org \\ ${ }^{2}$ Bulgarian Modeling\&Simulation Association - BULSIM, Sofia, Bulgaria \\ nikolay.tomov@bulsim.org \\ ${ }^{3}$ Space Research and Technology Institute, Bulgarian Academy of Science, Sofia, Bulgaria \\ irena.nikolova@mail.space.bas.bg
}

\begin{abstract}
This paper addresses the efforts of NATO, EU and other regional initiatives to support education and training for better understanding the Crisis and Disasters Management processes (CDMP) and Climate Change Implications (CCI) on Military activities. The article tackles the question of applying Modeling and Simulation systems, tools, software and practises as a complex combination of live, virtual, and constructive environment not only to predict the trends, but to identify the source in a particular situation. Currently in NATO (according the Action plan on $M \& S$ in support of military training) there is a significant gap in estimation, forecast, and response plan generation capabilities related to crisis and disaster management and climate change implication. The development of a specific technical platform for CDMP \&CCI will provide NATO\&EU with a unique comprehensive training and analytical capability. This platform is expected to enable the support of large scale crisis management and disaster response distributed exercises and analysis with specific tools and simulations for different types of Crisis and Disasters situations. This would enhance the quality of training of the militaries and will provide a critical new capability for Allies.
\end{abstract}

Keywords: CAX, Crisis Management, Disaster response, Comprehensive Approach, NATO-EU Cooperation, Civil-Military Interoperability, Interagency cooperation, Multinational Exercise, Smart defence, Pooling and Sharing, Risk reduction. Technical architecture, Simulation.

\section{Introduction}

The responsibility for crisis management and disaster response differs for every nation and may involve various ministries and agencies. It is also a core task of the Alliance. Today, the Alliance is able to make decisions in crisis and emergency situations and to act under significant threats and time pressure. NATO develops capabilities to be prepared, on a case-by-case basis and by consensus, to contribute in effective crisis and disaster prevention. This enables the Alliance to engage actively in crisis management 
and disaster response referring to non-Article 5 for crisis response operations. The Alliance therefore encourages the joint training of military and civilian personnel for building trust and confidence.

NATO's role in crisis management and disaster response goes beyond military operations and includes crisis and disaster response operations against natural or manmade disasters. A crisis can be political, military, social or humanitarian, and therefore NATO Crisis Response System covers different resources and capabilities of dealing with diverse types of crises, including disaster response.

Based on the perception that military intervention alone cannot resolve a crisis or ensure recovery after disasters, the NATO Summit Declarations and the Strategic Concept emphasizes the need for NATO to enhance its contribution to the Comprehensive Approach to crisis management. The goal is to enhance the Alliance and Nations' civilian and military capabilities for crisis management and disaster response.

Virtual reality, artificial intelligence algorithms, simulation of alternatives based on different scenarios, add high potential to risk analysis and assessment. With the support of modeling and simulation, various conditions could be created for: a holistic analysis, further development of existing methodologies, models, infrastructure, validation of working hypotheses by means of creating the environment for designing, testing and managing architectures of different systems, building models of systems, testing of specific criteria, running process optimisation, experimentation, assisting informative decision-making, etc.

Design and development of a simulation environment for natural disaster risk assessment include the need for realistic visualization of the impact from the concrete natural disaster, visualization of the affected groups and their capabilities (reaction time, scope of the disaster and potential negative impact, available resources, etc.) and precision in forecasting. Additionally, presenting the effects and impact on the population and on the environment as a result of real-time decision-making is almost impossible to be executed with traditional means. A very important activity is the analysis of the cooperation among all responsible stakeholders, dealing with natural disaster risk assessment.

Applying modeling and simulation for the preparation of all-levels authorities in disaster management becomes one of the most effective methods for education and training, which allows, with the use of minimum resources, the evaluation of the current level of preparedness and readiness of the national and institutional forces and resources.

The challenges that the Integrated Natural Disaster Risk Assessment Information System faces create the need for new preparation methods aiming to ensure maximum results with the use of minimum resources. This imposes the adaptation of available forces and resources to work in a joint multinational environment consistent with the requirements for speed, precision and flexibility. Subsequently, the requirements towards the system, considering the work in a joint multinational environment, will continue to increase demanding full interoperability. New doctrines and procedures concerning risk assessment and the mitigation of the negative impact caused by natural disasters have to be developed alongside the new systematic preparedness programmes. 
Modeling and simulation in crisis management and disaster risk assessment is applied in order to recreate imaginary critical situations. Modeling and simulation are becoming even more popular nowadays given the current level of societal development and the penetration of modern technologies in all academic, applied and socio-economic areas. Modern technologies enable the recreation of the reality into virtual reality with training purposes. Virtual reality is used to recreate specific events (terrorist attack, natural disaster, social anomaly) which is to serve the users to assess and be prepared in case similar potential real events occur.

\section{Disasters Impact on People}

In recent years, an increase in the number and the magnitude of man-made or natural disasters has been recorded around the world and particularly in Bulgaria. An increase in the negative effects of disasters on the social relations, the economic growth and the sustainable development of the countries is also registered.

According to the type of hazard, disasters can be generally classified in the listed groups bellow (The Human Cost of Weather-Related Disasters 1995-2015). The report focus on hydrological, meteorological and climatological disasters, which are collectively known as weather-related disasters (see Fig. 1).

- Natural Hazards by Disasters type

- Geophysical

- Earthquake

- Mass movement (dry)

- Volcanic activity

- Hydrological

- Floods

- Landslides

- Wave actions

- Meteorological

- Storms

- Extreme temperatures

- Fog

- Climatological

- Drought

- Glacial lake outburst flood

- Wildfires

- Biological

- Epidemic

- Animal accidents

- Insect infections

- Extra-Terrestrial

- Impact

- Space weather 
- Human costs and impacts of weather related disasters

- Manmade disasters

- Climate Change Implications.

The human losses caused by weather-related disasters depend on multiple factors, including the type of hazard, its location, duration and the size and vulnerability of the population. The basic economic impacts, including homes and infrastructure damaged and destroyed, must also be considered. Other costs, including repairs, rehabilitation and rebuilding expenditure, plus lost productivity and increased poverty, are harder to quantify but nevertheless must be taken into account when analysing the overall economic burden of disasters.

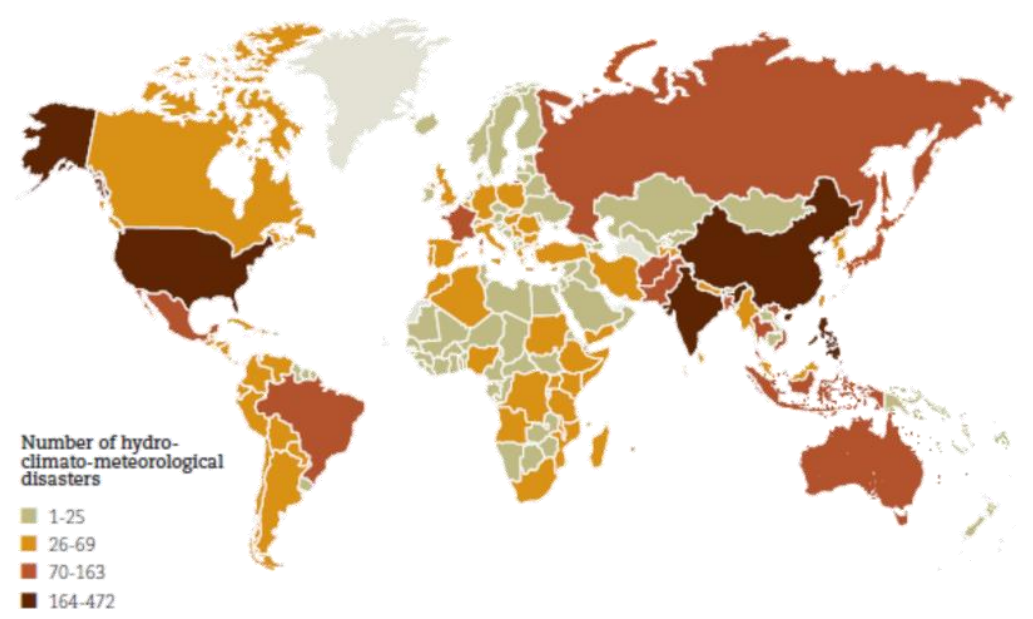

Fig. 1. Number of weather related disasters

The figure 2 shows that people are mainly affected by disasters such as flooding and drought.

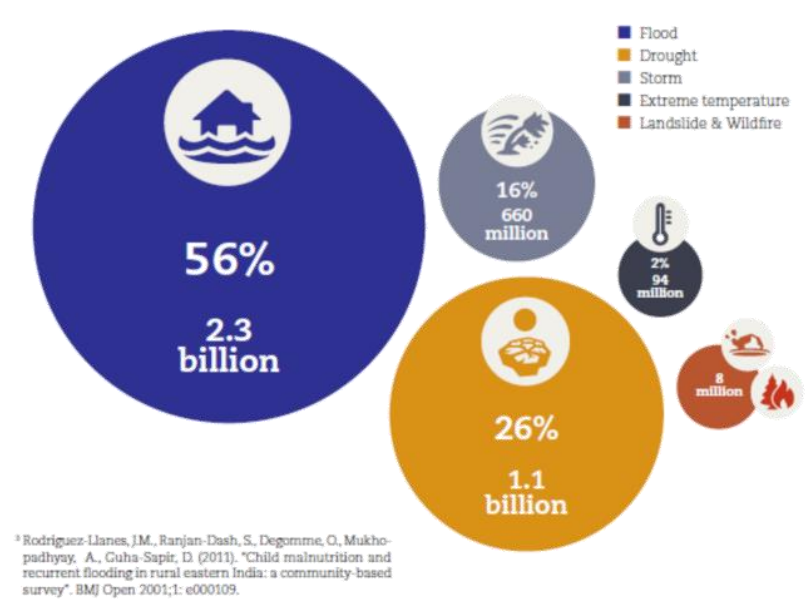

Fig. 2. Number of people affected by disasters 
When causes for deaths are analyzed the situation is completely different. Storms and Extreme temperature are main "killers". Storms, including hurricanes, cyclones and storm surges, killed more than 242,000 people between 1995 and 2015 (see Fig. 3), making storms the most deadly type of weather-related disaster in the last 21 years.

Scientific results suggest that climate change will increase the upward trend in the numbers of floods and storms worldwide, while the population that needs protection will increase at the same rate as population growth in disaster-prone regions. On the positive side, weather forecasting has made extraordinary progress in recent years, with predictions highly reliable within a 48-hour period. In the face of climate change, we may not be able to stem the increased frequency of storms, but better risk management and mitigation could reduce deaths and other heavy losses caused by those predictable hazards.

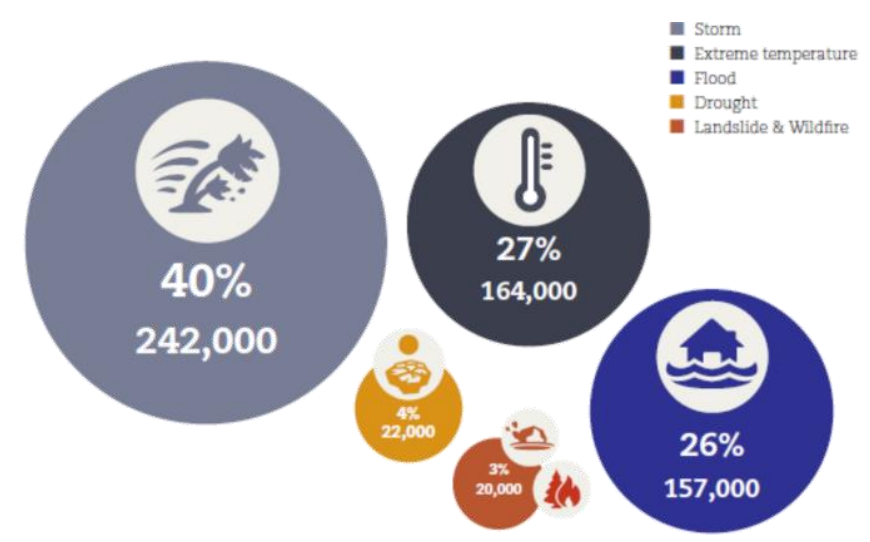

Fig. 3. Number of people killed in disasters type

\section{$3 \quad$ Modeling \& Simulation for NATO Crises Management and Disaster Response Support}

Managing crisis situations is a priority not only at national level, but also at NATO and EU level.

Crisis situations may entail large-scale incidents forcing the evacuation of civilian population and other drastic safety measures. Due to the scale of crisis situations a great number of managing bodies from different institutions are involved in the decisionmaking process. The actions taken by each responsible organisation - army force, police, civil protection, fire and medical units, as well as the voluntary units, have to be adequately coordinated and planned in advance in order to ensure rapid and effective response. To reach optimal effectiveness and efficiency in the actions undertaken, these forces have to be well-trained. The preparedness of the forces and the rapid allocation of resources during crisis situations cannot be achieved without regular training and exercises. 
At the same time, the rapid development of modern technologies has led to a broad application of modeling and simulation methodologies and tools in risk assessment, disaster management and training of teams and decision-makers. Simulation possesses high potential serves as a catalyst in the process of human capacity-building using innovations and experiments in training, evaluation of alternatives and validation of concepts, methodologies and procedures.

Their main purpose is to create, based on high-level architecture and technology, the specific conditions for individual training, coordination of actions at any level, as well as support decision-making on risk reduction and eliminate the impact of disasters.

The successful application of simulations in disaster risk assessment and the improvement in the preparation and qualification of staff members could enable various scenarios analyses, put the focus on preventive actions and enhance decision-making during natural disasters and emergencies. The analysed information allows higher precision in risk and impact assessment of emergency situations and increases the effectiveness of the rescue and recovery activities.

However, the capabilities of the CMDR Training and Climate Changes preparedness can be greatly enhanced by the utilization of the application of crisis management and disaster response tools, software and simulation systems.

The developed M\&S environment will have its initial operational capability (IOC) established and operational in time to support the planned NATO Crisis Management Exercises, as well as any regional Network projects or exercises and trainings.

The integration of all mentioned aspects will add value to NATO force structure in order to be well prepared for the next conflict by supporting capability building; improving interoperability and support of capability development with education and training for NATO and partner leaders and units; testing doctrines; developing and validating concepts through experimentation; providing lessons learned, evaluations and assessments.

\section{Integrated (Distributed) CDMP \&CCI Simulation Platform}

The combination of Live, Virtual and Constructive simulation is expected to form the Integrated CDMP \&CCI Simulation Platform. In the context of an architecture for a CMDR Simulation \& Training Environment we can identify the main "modules" of such a Platform as:

- Management Framework - the Management Framework includes the Integrated Management Cycle. Considering the specific training features and the best practices, the framework has been developed on the conceptual basis of the project management.

- Simulation Framework - application of a federated approach based on High Level Architecture (HLA), but could also include Distributed Interactive Simulation (DIS) and Test and Training Enabling Architecture (TENA);

- Modeling and Simulation Tools - tools including modeling and simulation of CBRN, flooding and earthquake effects during crises, crowd movement models and modeling of civil emergency services, etc.; 
- Environment Representation - including terrain, weather conditions and infrastructure;

- Training Audience with their C4I real tools including decision and situational assessment tools, also visualization, simulation control and analysis tools;

- ICT Infrastructure - including hardware, basic software and connectivity for supporting the functioning of the Training Environment.

These six main "modules" of the CDMP \&CCI Simulation Platform will enable a powerful Integrated (Distributed) Training Environment for CMDR.

Adapted to NATO's Concept Development and Experimentation Policy and processes for capability development the Integrated Environment for CMDR Training could be schematically presented as three main functional labs (see the figure bellow):

- Concept Development Lab - provides a framework for identifying existing ideas and solutions or developing new ones to overcome capability shortfalls and gaps in CDMP \&CCI;

- Experimentation Lab - its role is to determine, with the means and tools of experimentation, whether a concept under development will achieve its main aim;

- Training Lab - a place for exercising the approved concepts, tested and validated with experiments.

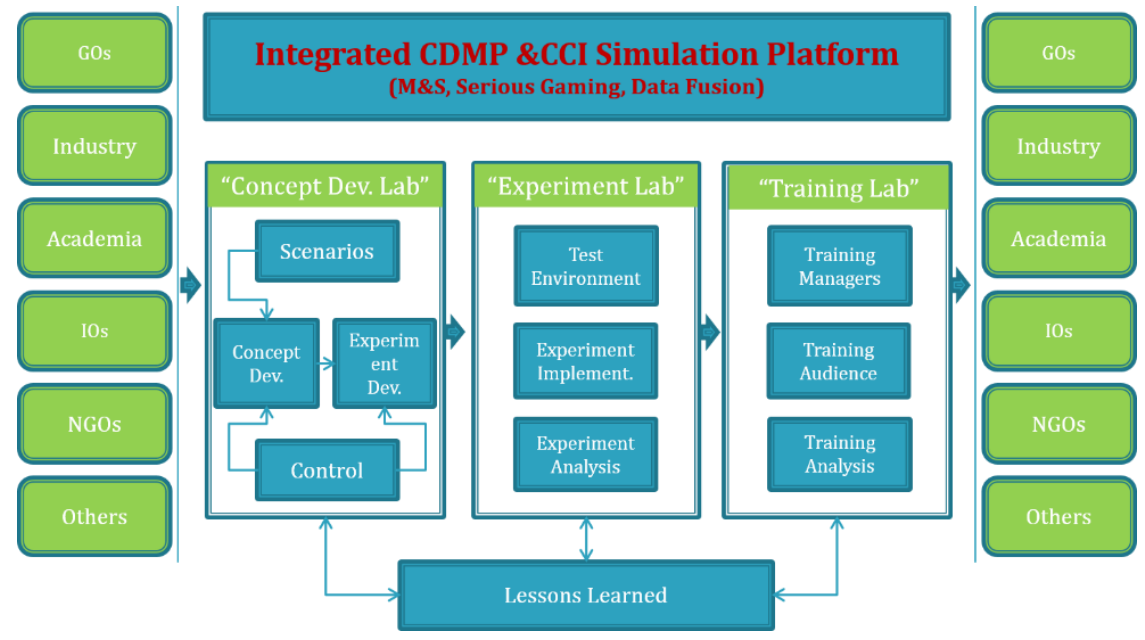

Fig. 4. CDMP \&CCI Simulation Platform

\section{Project Management of CDMP \&CCI Simulation Platform}

The realization of a CDMP \&CCI simulation platform from the management point of view is a complex activity which requires specific efforts in the area of the management and planning. In this regard, the Platform development should be planned and executed 
according to the Project Management Approach, using PRINCE2 as a specific organization dedicated to deliver its specific results/objectives.

PRINCE2 (PRojects IN Controlled Environments) is the most widely practiced project management methodology, based upon project management best practices and used in over 150 countries and NATO. PRINCE2 provides common systems, procedures and language.

The achievement of the objectives of the CDMP \&CCI simulation platform design, development and realization is ensured by good project management, which defines the exercise goals, organises the work packages and assigns the workload to highly motivated, qualified and experienced teams.

The specific benefits are as follows:

- CDMP \&CCI simulation platform implementation in scope, in time and in budget;

- Transparency, distinct distribution of responsibilities and assessment of deliverables and achieved results at each stage;

- More effective and efficient resource management;

- Storing data and knowledge bases.

\section{Trends in the Simulation Solutions for Disaster Risk Assessment}

Tendencies in the development of simulation solutions for disaster risk assessment are focused on the improvement of standards, services, communication, equipment and software, training, education and technical knowledge, necessary for the successful design and maintenance of a complex modeling and simulation program and for achieving maximum benefit from their utilisation.

A common technical environment enables the identification of standards for the use of modeling and simulation software which determines the technical architecture, including the type of interface of the additional programme applications.

The simulation architecture consists of main functional elements, interfaces and programming rules applicable to all simulation applications. It provides the general framework within which specific system architectures and higher-level architectures can be defined and made functional.

Data standards are used in creating, publishing and guiding the actions, procedures and the methodologies for defining the data requirements used in modeling and simulation. These requirements include data exchange, verification, validation and accreditation standards, and also standards for authorisation data sources and ensuring data security. Data standards improve the interoperability between modeling and simulation providing data and command, control, communication, computer and intelligence (C4I) systems. 


\section{$7 \quad$ Knowledge and Expertise in Simulation Development Technologies}

The acquisition and development of simulations with the aim of meeting a wide spectrum of users' needs is a very challenging task. The possibility of simulating exercises at any level, where the switching between different levels is performed in different simulation systems and simulators, reaching the demands of the individual training, is a highly complex task.

The usage of simulation systems ensuring Live Simulation, Virtual and Constructive Simulation brings to a qualitative new level the process of modeling and simulation. Each simulation is applied to different trainings, exercises and games. The 'Serious games' type will contribute to the specific procedures and missions of the training.

Technology evolves quickly and enables the increase in general effectiveness of the modeling and simulation. In order for the Integrated Natural Disaster Risk Assessment Information System to benefit from modern technologies, a constant research on their development has to be conducted. This process includes gaining knowledge on modeling and simulation, creating education database by referring to periodicals, participation in conferences, international programs, expert exchange and participation in international exercises conducted in a distributed manner.

\section{$8 \quad$ Remote Training and Distributed Exercises}

Conducting distributed CAX using a simulation module enables financial and material savings to be made as trainees will participate in the trainings from different locations. Effectively, the new joint capabilities enable the conduct of joint exercises run by various structures of different institutions and organisations without the need of physical presence at a specific location.

The goal is to establish a joint information system for training capabilities development, analysis and assessment using the entire spectrum of real, virtual and constructive simulations.

\section{Capability Analysis of Simulation Instruments for a Natural Disaster Risk Assessment}

Analysis conducted with the use of simulation provides the opportunity for almost comprehensive risk assessment, applying various probability and intelligent methods, with integrating diverse types of data and expertise in a context of subjectivity and incomplete certainty. Through simulation the theory easily turns into practice and enables people to use the pragmatic method in analysing and improving their work.

In order to create a 'Simulation' module as part of the Integrated Natural Disaster Risk Assessment Information System the necessary software and simulation systems have to be selected and to be connected into a common environment. The expectation that a single software can encompass all natural disasters is a more or less unachievable 
task considering the current state of the technologies. Globally, a number of simulation systems and software exist, which are designed to solve different tasks for anticipating and mitigating the risk of various natural and man-made disaster and for crises management in case such events occurred.

Exploring the simulation systems and software, with the aim of selecting the most appropriate combination for the purpose of training staff members in a close to the reality environment during a specific type of a natural disaster, and connecting them in a common environment in which different scenarios can be simulated, is a promising approach that could save time and resources.

The current practices shows that it is almost impossible, and unnecessary for that matter, to create a simulation model that includes all levels - from tactical to strategic. Overall, the trainees exercise different types of activities and it is not necessary for the high level authorities to be able to manage local finances, but rather to know what has to be the contribution for achieving a positive result.

Last but not least, attention should be paid to the fact that disasters are diverse and cyclic as well as differing from one geographic latitude to another. If, for the region of North America, typhoons and hurricanes are a widely spread disaster, for Europe these are floods, for Asia - earthquakes, and droughts for Africa.

The common trait of all these is that they are measured against material, financial and, almost in all cases, human life losses.

Due to the aforementioned reasons, to design and develop a stand-alone product for an integrated risk assessment covering all types of disasters is a heavy and unpractical task.

The integration of software products in a common environment is expected to contribute for tackling the aforementioned issue. Transferring scenarios, forces and resources, which may be observed on different simulation systems is an easier option for solving the generality issue of the task.

Solving this problem is imperative also because disasters know no borders and can easily spread from one country to another, and because the responsibilities for different disasters are shared by different ministries and institutions. Therefore, interagency cooperation and interaction is of crucial importance and simulation products provide the best result in training the management staff from different ministries in a realistic uniform environment.

\section{Criteria for Selection of Simulation Instruments and Solutions for Disaster Risk Assessment}

Well fitted simulation instruments suitable for risk assessment of various types of natural disasters on the territory of the Republic of Bulgaria - flood simulation, fire simulation, CBR contamination, evacuation simulation, etc. have been identified. The analysed simulation products and solutions, covering specific areas, were ranked in the following manner: 
- Selected simulation solutions applicable to the natural disaster risk assessment for the territory of the Republic of Bulgaria based on historical data on the frequency of occurrence of these and other anticipated disasters as a result of climate change;

- Selected simulation solutions on the basis of the criterion 'available open source solution information';

- Selected simulation solutions on the basis of 'information received from providers of simulation solutions';

- Selected simulation solutions on the basis of 'expertise'.

\section{NATO computer supported capabilities dealing with CDM\&CCI}

The 2015 Gap Analysis Report serves as the foundation for the development of the 2015 Action Plan (AP) on M\&S in support of military training. The Science, Technology, Modeling \& Simulation Branch NATO HQ SACT and the Crisis Management and Disaster Response Centre of Excellence (CMDR COE) staff realized that there is a gap in NATO computer supported capabilities dealing with large scale events with negative impact on human society such as crisis and disasters, as well as in the evaluation of Climate Change. CMDR COE's staff has started a project for developing a technical platform using $M \& S$ for conducting experiments, tests, CAXs and training in the CMDR and Climate Change domain.

The project was endorsed by the NATO Science and Technologies Board and started in February 2016 with an end date February 2019 under MSG-147 with lead nation Bulgaria and participation from Austria, Bulgaria, Germany, Sweden, USA, M\&S CoE, CMDR CoE, JFTC, and companies as IBM, MASA Group, C4IS. The project is open to Industry and NATO Partnering Nations.

The aim of the project is to develop a reference architecture and demonstrate a technical platform that enables prompt, reasonable and effective verification and validation of Crisis/Disaster and Climate Change Implication (CCI) Response plans.

The development includes scientific researches, concept development and experimentation, standardization and interoperability improvements.

The expected Deliverables from the projects:

- Investigate, categorize and catalogue data sources available for Disaster Response.

- Analyze existing standards in common vocabulary and adapt existing ones for NATO purposes.

- Categorize existing M\&S solutions for CMDR\&CCI and develop missing ones.

- Computer formalization of Command and Control logic for Crisis/Disaster Response (DR).

- Reference architecture/platform for proof of concept.

- Demonstration (contribution to multinational exercises) as a test bed for Analysis and Trainings in order to support Crisis/Disaster Management and Climate Change Implications.

- Synergies and Complementarities 
The project combines NATO Crisis Response Process with industrial theory for system control in predefined parameters. The task is of significantly large scale and is divided into subtasks and spread among NATO Nations, bodies, organizations and partners.

The three phases of the project will be synchronized to move forward at the most rapid pace possible.

Phase 1 is planned to take 12 months and will result in the development of a Master Plan to guide and direct this project. It would also identify all the requirements, products and specifications for support of the crisis management and disaster response technical platform for M\&S.

Phase 2 is planned to take twelve months and will result in building the initial operational capability (IOC) of the crisis management and disaster response technical platform. Planning of the experiments, verification and training for CMXs would be conducted as well during this phase.

Phase 3 is planned to take twelve months aiming to achieve successful execution of $\mathrm{CM}$ exercises and analysis efforts as part of the operational support of the crisis management and disaster response technical platform. During this phase, experienced subject matter experts will support the improvement of the platform. They would also conduct advance training for using the fielded simulations and tools.

The technology matureness has reciprocal effect to the command and control logic. Implementation of such developed technical products in NATO Crisis and Disaster Management process will smarten up it and that is one of the aims of MSG-147.

The project has three main directions for analyzing CDM in NATO in order to improve the E\&T and support of the decision making process in the Alliance:

- First one is the analysis of the Disaster Risk Management (DRM) processes, preceding the development of the Operations Plan:

- Fast and accurate Disaster Risk Analysis;

○ Comprehensive approach and correlation assessment among hazardous events;

- Prevention and Preparedness Measures proposals.

- Second one is the assessment of the Disaster Response, during NATO operation:

- Fast and accurate Disaster Assessment (DA);

○ Dynamically generated proposal for Response Plan.

○ Lessons Learned process

- Third one is the development of module for a realistic modeling and representation of different types of disasters for the purpose of education and training, experimentations, verification and validations.

The following components should be developed in order to achieve the above mentioned results:

- Input Data Module - database for statistical (history) and real-time data. The database should have standard properties and interface. 
- Disaster Model Engine - combines input interface (accept data from the database), disaster model repository (as many as possible mathematical representations of different types disasters), output interface.

- Decision-Making Support Module - database with fragmented SOPs and defined triggers for disaster alerts and response. To each elementary action of the SOPs, will be added metadata containing: relevance to event, priority and sequence, dependences, etc.

- Filtering and Distribution Module - will filter the relevant elementary response actions and will dynamically generate proposal for Response Plan or Prevention and Preparedness Measures proposal.

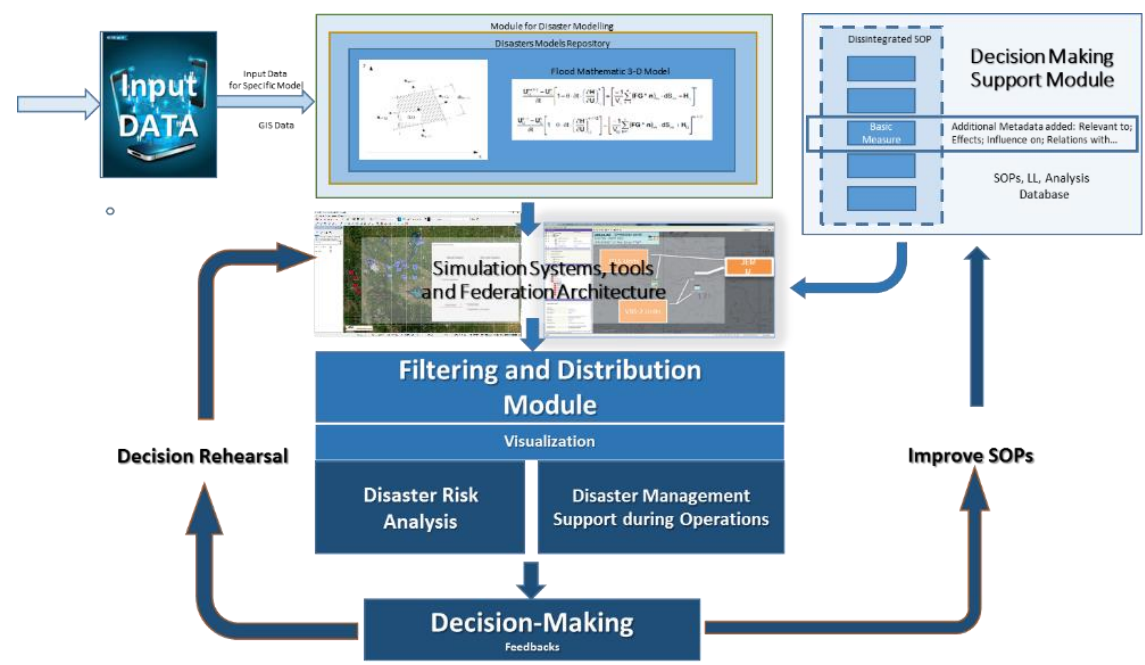

Fig. 5. Technical Architecture for CDMP \&CCI

\section{Conclusions}

The development of M\&S architecture specific for the needs of the CMDR Training and Climate Change preparedness would provide NATO with a unique comprehensive training and analytical capability for non-military type of operations. This M\&S architecture would be able to support large scale CDM distributed exercises and analysis with specific crisis management and disaster response tools and simulations. Experienced and highly trained personnel will operate this newly established technical environment. This new crisis management and disaster response environment will be supported by operationally experienced simulation subject matter experts to ensure successful operations, exercises and support activities from the beginning of the operations. 
The close cooperation in the crisis management and disaster response domain requires the involvement of the appropriate military and civilian capabilities. These capabilities will include information and intelligence sharing, developing and operating early warning systems (in support of building common situational awareness), as well as conducting crisis and disaster planning and response, and preparedness for Climate Changes.

The establishment of a CMDR M\&S Platform should have its initial operational capability established and operational in time to support the planned NATO Crisis Management Exercises, as well as any regional Network projects or exercises and trainings.

\section{References}

1. Nachev, M., Nikolov, O.: Multinational Initiatives and Training in Support of Regional Defense Cooperation - BG (2015).

2. Technical report MSG 068 "NATO Education and Training Network"

3. ACT Directive for Operating JWC, JFTC and JALLC (80-3), Version: Latest (March 2004).

4. ACT Directive for the Implementation of JWC, JFTC and JALLC Plan of Action and Milestones (80-6), Version: Latest (December 2004).

5. Provide Joint Training, Experimentation and Interoperability Development Capabilities (CP 9B0401), Version: Latest (June 2004).

6. JWC and JFTC Training and Experimentation Facility AIS Concept User Requirements Analysis, Version: 1.1 (December 2005).

7. BI-SC 75-3 Collective training and Exercise Directive, Version: Latest, OKT (2010).

8. MSG-068 NETN TAP, Version: Latest (April 2007).

9. STANAG 4603.

10. IEEE Standard 1516-2010 (2010).

11. Huiskamp, W., Wymenga, R., Krijnen, R. and Harmsen, E., Network Infrastructure Design Document for NATO Education and Training Network (NETN), (2009).

12. JFTC Presentation to SEEETN Balkan Bridges MPC - Charles M. Stibrany, CIV US Program Manager; Gultekin Arabaci CIV TU.

13. Nikolov, O.: Training for success. Joint Training initiatives improve security in the Balkans - Per Concordiam 6 (1) (2015).

14. Integrated Environment for Crises Management and Disaster Response Training, CMDR CoE Proceedings 1, Sofia (2015).

15. The human cost of weather related disasters report - Centre of research of epidemiology of disasters (CRED) (2015). 